\title{
ON A PROPERTY OF CASTELNUOVO VARIETIES
}

\author{
CIRO CILIBERTO
}

\begin{abstract}
Castelnuovo varieties are those irreducible complete varieties in a projective space whose geometric genus is maximal according to their dimension, degree and embedding dimension. In this paper, extending results by Severi and Accola, we prove that, under suitable conditions, such varieties are birational if and only if they are projectively equivalent.
\end{abstract}

Introduction. A very old result, already known to M. Noether, claims that two smooth plane curves of degree $n \geq 4$ are birational if and only if they are projectively equivalent. In the paper $[\mathbf{S}]$ of 1933 , Severi gave a very nice extension of this theorem to hypersurfaces in a projective space. More recently Accola proved in $[\mathbf{A}]$ an analogous property for special Castelnuovo curves. This paper deals with a further extension of these results. In fact both, Castelnuovo curves and hypersurfaces, are contained in the larger class of Castelnuovo varieties: these varieties, introduced and studied by Harris in $[\mathbf{H}]$, enjoy the property of having maximal geometric genus according to their dimension, degree and embedding dimension. Thus the theorems of Severi and Accola suggest to look for a more general statement of the same type concerning Castelnuovo varieties. This result can in fact be established (see Theorem 3.1.1) and $\S 3$ of this paper is devoted to its proof. Conceptually this proof is not so different from Severi's one, but is technically more entangled. In particular it requires some preliminary result about Castelnuovo varieties, for which, among other things, we add some detail to Harris' classification: this is the object of $\S \S 1$ and 2 .

Notation and conventions. We shall work over an algebraically closed field $K$ of characteristic zero. If $V$ is a $K$-scheme we shall denote by $p_{a}(V)$ its arithmetic genus and by $\omega_{V}$ its dualizing sheaf. If $\omega_{V}$ is invertible, a corresponding divisor will be denoted by $K_{V}$. If $V$ is smooth of dimension $k, \Omega_{V}^{k}$ will be, as usual, the sheaf of regular $k$-forms on $V$. Thus $\omega_{V}=\Omega_{V}^{k}$. If $\mathcal{F}$ is any coherent sheaf on $V$, we shall denote by $h^{i}(V, \mathcal{F})$ the dimension of the cohomology space $H^{i}(V, \mathcal{F})$ as a $K$-vector space. $\operatorname{Pic}(V)$ will be the Picard group of $V$ and $\sim$ the linear equivalence of divisors. If $V$ is smooth and $A, B$ are cycles of $V, A \cdot B$ will be their product in the Chow ring of $V$. If $D$ is a divisor of $V,|D|$ will denote the complete linear system of $D$ on $V$. Finally, if $V$ is a smooth curve, we shall denote, as usual, by $g_{n}^{r}$ a linear series of degree $n$ and dimension $r$ on $V$.

Received by the editors January 31, 1986 and, in revised form, September 5, 1986.

1980 Mathematics Subject Classification (1985 Revision). Primary 14C22, 14E05, 14M99.

Supported by C. N. R. and Ministero Pubblica Istruzione. 


\section{Definition of Castelnuovo varieties and remarks on Castelnuovo curves.}

1.1. Let $V \subset \mathbf{P}^{r}, r \geq 2$, be a complete, irreducible, nondegenerate variety of dimension $k \geq 1$ and degree $n$, and let $p: \tilde{V} \rightarrow V$ be a resolution of its singularities.

The geometric genus $g=h^{0}\left(\tilde{V}, \Omega_{\tilde{V}}^{k}\right)$ is independent of the resolution and is therefore called the geometric genus of $V$. There is an upper bound for $g$, depending on $n, r, k$, discovered by Castelnuovo, for $k=1$, and Harris, for $k \geq 2$ (see [H]). If $n-1=M(r-k)+e$, with $e=0, \ldots, r-k-1$, then the Castelnuovo-Harris bound is

$$
g=g(n, r, k)=\left(\begin{array}{c}
M \\
k+1
\end{array}\right)(r-k)+\left(\begin{array}{c}
M \\
k
\end{array}\right) e .
$$

Let

$$
\chi= \begin{cases}M, & \text { if } e>0, \\ M-1, & \text { if } e=0 .\end{cases}
$$

Thus $g(n, r, k)>0$ if and only if $\chi \geq k$ or, equivalently, if $n \geq k(r-k)+2$. Assuming, as we shall do from now on, that this happens, $V$ is called a Castelnuovo variety if the equality holds in (1.1.1). For all properties of Castelnuovo varieties which will not be proved here, we refer to $[\mathbf{H}]$.

1.2. If $k=1, V$ is a projectively normal, hence nonsingular, curve. The equality $\chi=1$, equivalent to $n \geq 2 g+1$, characterizes nonspecial Castelnuovo curves. Let $\chi \geq 2$ or, equivalently, $n \geq 2 r$. The equality $n=2 r$ holds if and only if $V$ is a canonical curve. Finally a lemma of projective geometry (see $[\mathbf{H}$, p. 36]) gives a clear picture of the situation if $n \geq 2 r+1$. This lemma implies that either $r=2$, or $V$ lies on an irreducible surface $F$ of degree $r-1$ in $\mathbf{P}^{r}$, cut out by all quadrics containing $V$. Nondegenerate surfaces of degree $r-1$ in $\mathbf{P}^{r}$ are well known: they are either rational normal scrolls or the Veronese surface in $\mathbf{P}^{5}$. The problem of classifying Castelnuovo curves of degree $n \geq 2 r+1, r \geq 3$, is then reduced to identify them among all divisors on these surfaces.

1.3. Let $F$ be a Veronese surface in $\mathbf{P}^{5}$ and $L$ a conic on it. Then $V \sim a L, n=2 a$, $g=\left(2_{2}^{a-1}\right)$, where

$$
a= \begin{cases}2 M+2, & \text { if } a \equiv 0(\bmod 2), \\ 2 M+1, & \text { if } a \neq \equiv 0(\bmod 2) .\end{cases}
$$

Computing $g(n, 5,1)$ one checks that any smooth curve in the linear system $|a L|$ is a Castelnuovo curve. The condition $n \geq 2 r+1$ is equivalent to $a \geq 6$.

(1.3.1) REMARK. $V$ is isomorphic to a smooth plane curve of degree $a$, embedded in $\mathbf{P}^{5}$ via the complete linear series cut out on it by conics. Therefore, for any $g_{m}^{1}$ on $V$, one has $m \geq a-1$.

1.4. If $F$ is a scroll, let $f: S \rightarrow F$ be a minimal resolution of the singularities of $F: f$ is not an isomorphism if and only if $F$ is a cone over a rational normal curve. $\operatorname{Pic}(S)$ is freely generated over $\mathbf{Z}$ by the divisors $H$ and $R$, strict transforms via $f$ of the general hyperplane section and of a line of the ruling of $F$ respectively. Identifying $V$ with its strict transform on $S$, one has that $V$ is a Castelnuovo curve if and only if either

$$
V \sim(M+1) H+(r-2-e) R
$$


or, only if $e=0$,

$$
V \sim M H+R
$$

Since $K_{S} \sim-2 H+(r-3) R$, by adjunction one gets

$$
K_{V} \sim \begin{cases}(M-1) H+\left.(e-1) R\right|_{V}, & \text { if (1.4.1) holds, } \\ (M-2) H+\left.(r-2) R\right|_{V}, & \text { if (1.4.2) holds. }\end{cases}
$$

1.5. Some remarks on linear series on Castelnuovo curves on a scroll will be useful later on. The linear pencil $|R|$ cuts out a $g_{m}^{1}$ on $V$, with

$$
m= \begin{cases}M+1, & \text { if }(1.4 .1) \text { holds, } \\ M, & \text { if (1.4.2) holds. }\end{cases}
$$

This $g_{m}^{1}$ turns out to be base points free and complete. The following result is essentially due to Accola (see [A, p. 362]):

(1.5.1) THEOREM. Let $g_{t}^{1}$ be a base points free linear series on $V$. Then $t \geq \chi+1$. If either $e>0$ or $e=0$ and (1.4.2) holds, then $t=\chi+1$ if and only if $g_{t}^{1}=g_{m}^{1}$. If $e=0$ and (1.4.1) holds, then $t=\chi+1$ if and only if $r=3, F$ is a smooth quadric and $V$ is a curve of type $(M, M+1)$ on it; moreover $t=M+1=\chi+2$ if and only if $g_{t}^{1}=g_{m}^{1}$.

A slight extension of this theorem will be needed in what follows. The linear series $\left|K_{V}-(\chi-1) H\right|_{V} \mid$ on $V$ is a $g_{T}^{D}$ equal either to $|(e-1) R|_{V} \mid$ if $e>0$, or to $|(r-2) R|_{V} \mid$ if $e=0$ and (1.4.2) holds, or to $|(H-R)|_{V} \mid$ if $e=0$ and (1.4.1) holds. In any case

$$
D+1=h^{1}\left(V, O_{V}((\chi-1) H)\right)= \begin{cases}e, & \text { if } e>0 \\ r-1, & \text { if } e=0\end{cases}
$$

while, by (1.4.3), we have

$$
T=S(\chi+1)= \begin{cases}(e-1)(\chi+1), & \text { if } e>0, \\ (r-2)(\chi+1), & \text { if } e=0 .\end{cases}
$$

We notice that the $g_{T}^{D}$ is composite with the $g_{m}^{1}$ unless $g_{T}^{D}=|(H-R)|_{V} \mid$, in which case either $r=3$ and $g_{T}^{D}=g_{M}^{1}$, or $r>3$ and $g_{T}^{D}$ is not composite. In fact $|(H-R)|_{V} \mid$ is cut out on $V$ by the hyperplanes containing a line $l$ of the ruling of $F$, off $l \cap V$. If $|(H-R)|_{V} \mid$ is composite, then any hyperplane of $\mathbf{P}^{r}$ through $l$ and a general point $p$ of $V$ contains another point $q$ of $V$. Therefore the line $p q$ intersects $l$. Since $F$ is cut out by quadrics, $p q$ lies on $F$, thus $F$ has two rulings of lines, so is a quadric in $\mathbf{P}^{3}$.

We can now prove the

(1.5.2) THEOREM. Given any base points free $g_{t}^{d}$ on $V$ such that $d \geq D$, then $t \geq T$, and $t=T$ if and only if $g_{t}^{d}=g_{T}^{D}$.

PROOF. We may assume the $g_{t}^{d}$ to be complete. If it is composite of a rational involution, then the assertion follows by Theorem (1.5.1). If $g_{t}^{d}$ is not composite of a rational involution, it is possible to estimate the number $t-d$ of independent conditions which the general divisor of $g_{t}^{d}$ imposes to $\left|K_{V}\right|$, by means of a wellknown method by Castelnuovo (see [A, p. 363 and Theorem 4.1]). If $g_{t}^{d} \neq g_{T}^{D}$ one finds $t-d \geq S \chi+1$, whence $t \geq T+1$. 


\section{Some properties of Castelnuovo varieties.}

2.1. We consider now Castelnuovo varieties of dimension $k \geq 2$. One of the main properties of a Castelnuovo variety is that its general hyperplane section is again a Castelnuovo variety, thus the classification is worked out inductively.

Since $\chi \geq k \geq 2$, the general curve section $C$ of $V$ with a $\mathbf{P}^{r-k+1}$ is a special Castelnuovo curve. $C$ is a canonical curve if and only if $n=2(r-k+1)$, and this may only happen if $k=2$, in which case $V$ is a $K 3$ surface in $\mathbf{P}^{r}$. Let now $n>2(r-k+1)$. If $k=r-1, V$ is a hypersurface of degree $n \geq r+1$ in $\mathbf{P}^{r}$. If $k<r-1$, by the projective normality of $V$ and the properties of its general curve section, it turns out that $V$ lies on an irreducible, nondegenerate variety of dimension $k+1$ and degree $r-k$ in $\mathbf{P}^{r}$, which is cut out by all quadrics containing $V$. Varieties $F$ of this type are called minimal varieties.

2.2. In order to classify Castelnuovo varieties, one has to understand first minimal varieties. Luckily enough these are well known (see $[\mathbf{H}, \S 3]$ ), and turn out to be rational, projectively normal, of the following three types:

(2.2.1) rational normal scrolls $F_{a_{1}, \ldots, a_{k}}=\mathbf{P}\left(\mathcal{O}_{\mathbf{P}^{1}}\left(-a_{1}\right) \oplus \cdots \oplus \mathcal{O}_{\mathbf{P}^{1}}\left(-a_{k}\right)\right)$, with $0 \leq a_{1} \leq \cdots \leq a_{k}$ and $\sum_{i=1}^{k} a_{i}=r-k$

(2.2.2) quadric hypersurfaces in $\mathbf{P}^{r}$, if and only if $k=r-2$;

(2.2.3) cones projecting the Veronese surface of $\mathbf{P}^{5}$ from a general $\mathbf{P}^{r-6}$ of $\mathbf{P}^{r}$, only if $r \geq 6$ and $k=r-4$; we shall call these varieties Veronese cones.

If $F$ is of type (2.2.1), it is singular if and only if it is a cone over a scroll of lower dimension. Precisely if $a_{1}=\cdots=a_{i+1}=0$, then $F_{a_{1}, \ldots, a_{k}}$ is a cone over $F_{a_{i+2, \ldots}, a_{k}}$ from a $\mathbf{P}^{i}$, and these are the only cases in which $F_{a_{1}, \ldots, a_{k}}$ is singular. In particular $F_{0, \ldots, 2}$ is a rank 3 quadric, while $F_{0, \ldots, 0,1,1}$ is a rank 4 one. A resolution $f: S \rightarrow F$ of the singularities of $F$ can be obtained by blowing up the vertex of the cone in $\mathbf{P}^{r}$. We denote by $H, R, E$ the strict transform via $f$ of a general hyperplane section of $F$, of a general $\mathbf{P}^{k}$ of the ruling, the exceptional divisor corresponding to the vertex of the cone respectively. Then the following formula holds for the canonical system $K_{S}$ of $S$ :

$$
K_{S} \sim-(k+1) H+(r-k-2) R+(k-i-1) E
$$

where $i$ is the dimension of the singular locus of $F$. Formula (2.2.4) is proved in [H] in case $F$ is nonsingular. The case $i \geq 0$ can be worked out in a similar manner.

If $F$ is a quadric hypersurface, we may assume its rank $\rho$ to be 5 or more, otherwise $F$ is of type (2.2.1). As above a resolution $f: S \rightarrow F$ of $F$ may be obtained by blowing up the vertex of $F$, which is a $\mathbf{P}^{r-\rho}$. Giving $H, E$ the same meaning as above, one has the formula

$$
K_{S} \sim-(r-1) H+(\rho-3) E .
$$

Finally let $F$ be a Veronese cone. Consider a resolution $f: S \rightarrow F$ obtained in the usual way and give $H, E$ the usual meaning. Moreover denote by $L$ the strict transform via $f$ of a rank 3 cone on $F$. Then one has

$$
K_{S} \sim-(r-4) H-L \text {. }
$$

Formulas (2.2.5) and (2.2.6) can be found in $[\mathbf{H}]$.

2.3. Let us examine the case the Castelnuovo variety is contained in a scroll $F$. We denote by $W$ the strict transform of $V$ on $S$ via $f$. Then $W \sim a H+b R+c E$, 
with $a, b, c$ integers. If $F$ is smooth, $F=S$ and $E=0$, thus $c$ may be assumed to be 0 . If $i=k-1$, then $\operatorname{Pic}(S)$ is freely generated by $H$ and $R$ over $\mathbf{Z}$, and again we may assume $c=0$. Looking at the general curve section of $V$, and applying results of $\S 1.4$, one finds either $a=M+1, b=-(r-k-1-e)$, or, only if $e=0$, $a=M, b=1$. On the other hand, if $i=0, \ldots, k-2$, one has $c \leq 0$, because $W$ is irreducible. Let us now show that

(2.3.1) LEMMA. If $i=0, \ldots, k-2$, then

$$
c+(k-i-1) \geq \begin{cases}-1, & \text { if } e=0, a=M+1, \\ 0, & \text { otherwise. }\end{cases}
$$

Proof. Let $g: W^{\prime} \rightarrow W$ be a normalization. Then $h^{0}\left(W^{\prime}, \omega_{W^{\prime}}\right) \leq h^{0}\left(W, \omega_{W}\right)$ (see [HR, Ex. 6.10, p. 239, Ex. 7.2, p. 249]). Moreover, if $h: \tilde{V} \rightarrow W^{\prime}$ is a resolution of the singularities of $W^{\prime}$, by a result of Kunz (see [K, p. 105]), we have

$$
g(n, r, k)=g=h^{0}\left(\tilde{V}, \omega_{\tilde{V}}\right) \leq h^{0}\left(W^{\prime}, \omega_{W^{\prime}}\right) \leq h^{0}\left(W, \omega_{W}\right) .
$$

The dualizing sheaf $\omega_{W}$ is invertible and precisely $\omega_{W}=O_{W}\left(W+K_{S}\right)$. Since $h^{0}\left(S, \omega_{S}\right)=h^{1}\left(S, \omega_{S}\right)=0$, we get $h^{0}\left(W, \omega_{W}\right)=h^{0}\left(S, \omega_{S}(W)\right)$. It is now easy to see, using formula (2.2.4), that if the assertion were not true, then

$$
h^{0}\left(W, \omega_{W}\right)<h^{0}\left(S, O_{S}((a-k-1) H+(b+r-k-2) R)\right)
$$

but the right-hand side of this inequality can be easily computed (see $[\mathbf{H}, \S 4]$ ), and turns out to be equal to $g(n, r, k)$. This gives a contradiction.

(2.3.2) REMARK. The proof of Lemma (2.3.1) shows more than stated. In fact, $V$ being normal, we find $h^{0}\left(W, \omega_{W}\right) \leq h^{0}\left(V, \omega_{V}\right)=h^{k}\left(V, O_{V}\right)=p_{a}(V)$, the last equality holding because $V$ is projectively normal. Now $p_{a}(V)$ can be easily calculated by computing $h^{0}\left(W, O_{W}(l H)\right), l \gg 0$, and it turns out that $p_{a}(V)=g(n, r, k)$. Therefore $g(n, r, k)=h^{0}\left(\tilde{V}, \omega_{\tilde{V}}\right)=h^{0}\left(W^{\prime}, \omega_{W^{\prime}}\right)=h^{0}\left(W, \omega_{W}\right)=h^{0}\left(V, \omega_{V}\right)=$ $p_{a}(V)$, and then $p: \tilde{V} \rightarrow V$ induces an isomorphism $p^{*}: H^{0}\left(V, \omega_{V}\right) \rightarrow H^{0}\left(\tilde{V}, \omega_{\tilde{V}}\right)$. Let $U$ be an open subset of smooth points of $V$, such that $\operatorname{dim}(V-U) \leq k-2$. Then $H^{0}\left(U, \Omega_{U}^{k}\right) \simeq H^{0}\left(V, \omega_{V}\right)$ by the quoted result of Kunz. Furthermore, since $p$ is an isomorphism over $U, U$ can be seen as an open subset of $\tilde{V}$. Therefore the inclusion $U \rightarrow \tilde{V}$ yields an isomorphism $H^{0}\left(U, \Omega_{U}^{k}\right) \simeq\left(\tilde{V}, \Omega_{\tilde{V}}^{k}\right)$. It is perhaps worthwhile to observe that $\omega_{V}$ is locally free and generated by its global sections away from the vertex of the cone $F$. So, except the singularities $V$ has there, $V$ has only canonical singularities, in the sense of $M$. Reid (see $[\mathbf{R}]$ ).

Summing up, if $V$ is as above, then either

$$
\begin{aligned}
& W \sim(M+1) H-(r-k-1-e) R+c E, \quad 0 \geq c \geq i-k, \\
& K_{W} \sim(M-k) H+(e-1) R+\left.(c+k-i-1) E\right|_{W},
\end{aligned}
$$

the equality $c=i-k$ being possible only if $e=0$, or

$$
\begin{aligned}
& W \sim M H+R+c E, \quad 0 \geq c \geq i-k+1 \\
& K_{W} \sim(M-k-1) H+(r-k-1) R+\left.(c+k-i-1) E\right|_{W}
\end{aligned}
$$

only if $e=0$.

2.4. Let $V$ be contained in a quadric $F$ of rank $\rho \geq 5$. Then using analogous notation as before, by $(2.2 .5)$ we get

$$
W \sim n H / 2+c E, \quad K_{W} \sim(n / 2-r+1) H+\left.(c+\rho-3) E\right|_{W},
$$

where $n \equiv 0(\bmod 2)$ and $c \leq 0$. With the same proof of $(2.3 .1)$ one may show that 
(2.4.2) LEMMA. $c+\rho-3 \geq 0$.

Also Remark (2.3.2) can be repeated without changes.

2.5. Finally we suppose $V$ contained in a Veronese cone $F$, and use the same notation as above. $\operatorname{Pic}(S)$ is generated over $\mathbf{Z}$ by $H$ and $L$, and $E \sim H-2 L$. Thus

$$
\begin{aligned}
W & \sim a H+\frac{n-4 a}{2} L, \\
K_{W} & \sim(a-r+4) H+\left.\frac{n-4 a-2}{2} L\right|_{W},
\end{aligned}
$$

where $a \geq 0$, and (2.2.6) has been used.

(2.5.2) Lemma. One has $n \equiv 0(\bmod 2)$. If $n \equiv 0(\bmod 4)$, then either $a=n / 4$ or $a=n / 4-1$. If $n \not \equiv 0(\bmod 4)$ then $a=(n-2) / 4$.

PROOF. By (2.5.1) with standard calculation we get

$h^{0}\left(W, \omega_{W}\right)=4\left(\begin{array}{l}a-1 \\ r-3\end{array}\right)+(3+n-4 a)\left(\begin{array}{l}a-1 \\ r-4\end{array}\right)+\frac{(n-4 a)(n-4 a+2)}{8}\left(\begin{array}{l}a-1 \\ r-5\end{array}\right)$.

Further, the same argument of the proof of (2.3.1) implies that

$$
g=g(n, r, k) \leq h^{0}\left(W, \Omega_{W}\right) .
$$

If $n=4 b$, we must have $b \geq a$, otherwise $W$ would not be irreducible. Computing $g(n, r, k)$ one has

$$
g(n, r, k)=4\left(\begin{array}{l}
b-1 \\
r-3
\end{array}\right)+3\left(\begin{array}{l}
b-1 \\
r-4
\end{array}\right)
$$

and by (2.5.3) and (2.5.4) we have either $a=b$ or $a+1=b$. The proof is similar if $n \not \equiv 0(\bmod 4)$.

(2.5.5) REMARK. If $n \equiv 0(\bmod 4)$, then $V$ is a complete intersection of the Veronese cone with a hypersurface of degree $n / 4$, but in the case $a=n / 4$ the hypersurface does not pass through the vertex of $F$, while if $a=n / 4-1$ it does. It is perhaps worthwhile to explicitly point out that the singularity $V$ acquires in this case is not canonical, although it does not affect the geometric genus. A natural question would be to classify all singularities for which this happens.

2.6. Before going further, we summarize the classification of Castelnuovo varieties. It is natural to call nonspecial Castelnuovo varieties those for which $\chi=1$, special all the other ones. For the latter we distinguish between ordinary ones, for which $n>2(r-k+1)$, and exceptional ones, for which $n=2(r-k+1)$. The classification is given by the following

(2.6.1) THEOREM. Let $V \subset \mathrm{P}^{r}$ be a complete, nondegenerate Castelnuovo variety of dimension $k$ and degree $n$. Then:

(a) $V$ is nonspecial if and only if it is a smooth, nonspecial curve of degree $n \leq 2 r-1$ and genus $g=n-r$;

(b) $V$ is exceptional if and only if it is:

$\left(b_{1}\right)$ either a smooth canonical curve of degree $n=2 r$;

$\left(\mathrm{b}_{2}\right)$ or a $K 3$ surface of degree $n=2(r-1)$; 
(c) $V$ is ordinary if and only if it is of one of the following types:

(c $\left.c_{1}\right)$ a hypersurface of degree $n \geq r+1(n \geq 5$ if $r=3)$, with canonical singularities;

$\left(c_{2}\right)$ a variety of codimension one in a rational normal scroll $F$; if $F$ has a $\mathbf{P}^{i}$ of singular points, then:

$\left(c_{2,1}\right)$ either $V$ is residual to $r-k-1-e \mathbf{P}^{k}$ 's of $F$ in a complete intersection with a hypersurface of degree $M+1$, passing with multiplicity $m \leq k-i-1$ if $e>0$, $m \leq k-i$ if $e=0$, through $\mathbf{P}^{i}$, and $V$ has canonical singularities away from $\mathbf{P}^{i}$;

$\left(c_{2,2}\right)$ or $V$ minus a $\mathbf{P}^{k}$ is homologous on $F$ to a complete intersection with a hypersurface of degree $M$, passing with multiplicity $m \leq k-i-1$ through $\mathbf{P}^{i}$, and $V$ has canonical singularities away from $\mathbf{P}^{i}$;

$\left(c_{3}\right)$ a variety of codimension one in a quadric hypersurface $F$ of rank $\rho \geq 5$, complete intersection of $F$ with a hypersurface of $\mathbf{P}^{r}$ passing with multiplicity $m \leq$ $\rho-3$ through the vertex of $F$, and $V$ has canonical singularities away from this vertex; in this case $k=r-2$;

$\left(c_{4}\right)$ a variety of codimension one in a Veronese cone $F$, and precisely:

$\left(c_{4,1}\right)$ either a complete intersection of $F$ with a hypersurface of $\mathbf{P}^{r}$ passing with multiplicity $m \leq 1$ through the vertex of $F$, and such that $V$ has canonical singularities away from this vertex;

$\left(c_{4,2}\right)$ or $V$ minus a rank 3 quadric cone contained in $F$ is homologous on $F$ to a complete intersection of $F$ with a hypersurface of $\mathrm{P}^{r}$, and $V$ has canonical singularities away form the vertex of $F$; in case $\left(\mathrm{c}_{4}\right), k=r-4$.

This theorem has been proved for a great deal in $[\mathbf{H}]$. Here we have added cases $\left(b_{2}\right),\left(c_{1}\right),\left(c_{4,2}\right)$, escaped to Harris' classification, and have made more precise the statement if $F$ is singular in the ordinary case. It should be noted that in case $\left(c_{1}\right)$ the assertion about the singularities of $V$ follows by the same argument of (2.3.2).

2.7. In what follows we shall need the

(2.7.1) LEMMA. Let $V \subset \mathbf{P}^{r}$ be a Castelnuovo variety of dimension $k$ and degree $n$, such that

$$
n \geq(k+1)(r-k)+2 .
$$

Let $U$ be the nonsingular locus of $V$; looking at $U$ as an open subset of $\tilde{V}$, then the canonical map of $\tilde{V}$ is an isomorphism of $U$ onto its image.

PROOF. We examine only the nontrivial case $k \geq 2$. Since $n \geq(k+1)(r-k)+2$ implies $n>2(r-k+1), V$ is ordinary. If $k=r-1$, then $n \geq r+2$ and the assertion is clearly true. Let $V$ be of type $\left(c_{2}\right)$. The hypothesis implies

$$
M \geq \begin{cases}k+2, & \text { if } e=0, \\ k+1, & \text { if } e>0 .\end{cases}
$$

Thus the assertion follows by (2.3.3) and (2.3.4). If $V$ is of type $\left(c_{3}\right)$, then the hypothesis yields $n / 2 \geq r$, and by (2.4.1) we are done. Finally, if $V$ is of type $\left(c_{4}\right)$, then $n \geq 4 r-10$ and the lemma follows by (2.5.1) and Lemma (2.5.2).

If $n \leq(k+1)(r-k)+1$ it may very well happen that the canonical map of $V$ is not birational. It is not difficult to completely classify Castelnuovo varieties for which this happens. 


\section{An extension of a theorem of Severi.}

3.1. This section is devoted to prove the following extension of a theorem of Severi (see $[\mathbf{S}])$ :

(3.1.1) THEOREM. Let $V \subset \mathbf{P}^{r}$ be a Castelnuovo variety of dimension $k$ and degree $n$ such that $n \geq(k+1)(r-k)+2$. Let $V^{\prime} \subset \mathbf{P}^{s}$ be a nondegenerate, complete variety of dimension $k$, of degree $n^{\prime} \leq n$, such that $s \geq r$ and there is a rational dominant map $\pi: V^{\prime} \rightarrow V$. Then $r=s$ and $\pi$ is an isomorphism induced by an invertible linear transformation of $\mathbf{P}^{r}$.

The proof will be divided in several steps.

3.2. Step 1: $n=n^{\prime} r=s$ and $V^{\prime}$ is a Castelnuovo variety. Let $p: \tilde{V} \rightarrow$ $V, p^{\prime}: \tilde{V}^{\prime} \rightarrow V^{\prime}$ be resolutions of singularities. Then there exists a rational, dominant map $\tilde{\pi}: \tilde{V}^{\prime} \rightarrow \tilde{V}$ induced by $\pi$. Accordingly the linear map $\tilde{\pi}^{*}: H^{0}\left(\tilde{V}, \Omega_{\tilde{V}}^{k}\right) \rightarrow$ $H^{0}\left(\tilde{V}^{\prime}, \Omega_{\tilde{V}^{\prime}}^{k}\right)$ is injective. Whence

$$
g(n, r, k)=h^{0}\left(\tilde{V}, \Omega_{\tilde{V}}^{k}\right) \leq h^{0}\left(\tilde{V}^{\prime}, \Omega_{\tilde{V}^{\prime}}^{k}\right) \leq g\left(n^{\prime}, s, k\right) .
$$

Now it is easily checked that $g\left(n^{\prime}, s, k\right) \leq g(n, r, k)$, and the equality holds if and only if $n=n^{\prime}, r=s$. Thus the equality holds in (3.2.1), proving Step 1 .

3.3. Step 2. There is an open subset $U^{\prime}$ of $V^{\prime}$, consisting of smooth points of $V^{\prime}$, with $\operatorname{dim}\left(V^{\prime}-U^{\prime}\right) \leq k-2$, such that $\pi$ induces on $U^{\prime}$ a well-defined birational morphism $\tilde{\pi}: U^{\prime} \rightarrow \tilde{V}$. Since $V^{\prime}$ is normal, the existence of $U^{\prime}$ with the required properties, over which $\tilde{\pi}$ is well defined, is obvious. Notice now that $\tilde{\pi}^{*}: H^{0}\left(\tilde{V}, \Omega_{\tilde{V}}^{k}\right) \rightarrow H^{0}\left(U^{\prime}, \Omega_{U^{\prime}}^{k}\right)$ is an isomorphism (see $\S 3.2$ and $(2.3 .2)$ ), therefore $\tilde{\pi}$ is birational by Lemma (2.7.1).

Step 2 in particular implies that $\pi$ is birational, and in fact an isomorphism, if $k=1$. This proves the theorem for $k=1$, by a result of Accola (see $[\mathbf{A}]$ ). From now on we assume $k \geq 2$, and we shall denote by $\pi: U^{\prime} \rightarrow V$ the composite morphism $p \circ \tilde{\pi}$.

3.4. Step 3. Let $C^{\prime}$ be the general curve section of $V^{\prime}$ with a $\mathbf{P}^{r-k+1}$; we may assume $C^{\prime} \subset U^{\prime}$. Let $C=\pi\left(C^{\prime}\right)$; then the linear span of $C$ in $\mathbf{P}^{r}$ is a $\mathbf{P}^{r-k+l}$, with, $l \geq 1$. The first assertion is trivial. Let now $p_{1}^{\prime}, \ldots, p_{r-k+2}^{\prime}$ be general points on $C^{\prime}$. They are general points on $U^{\prime}$ too and the corresponding points $p_{1}, \ldots, p_{r-k+2}$ are still general points on $V$ belonging to $C$. Since $p_{1}, \ldots, p_{r-k+2}$ span a $\mathbf{P}^{r-k+1}$ contained in the linear span of $C$, the assertion follows.

(3.4.1) REMARK. With the above notation, $C^{\prime}$ is a Castelnuovo curve of degree $n$ in $\mathbf{P}^{r-k+1},\left.\pi\right|_{C^{\prime}}: C^{\prime} \rightarrow C$ is a birational morphism, $C$ is a curve of degree $m$ in $\mathbf{P}^{r-k+l}$ with $l \geq 1$. Since the theorem holds for $k=1$, we have $m \geq n$ and the equality holds if and only if $l=1, C$ is a Castelnuovo curve, $\left.\pi\right|_{C^{\prime}}$ is induced by an invertible linear transformation, and $C$ is the general curve section of $V$.

3.5. Step 4: $n=m$. Since $n \geq(k+1)(r-k)+2$, both $V, V^{\prime}$ are ordinary Castelnuovo varieties. Using notation introduced in $\S 2$, we shall denote by $W$, $W^{\prime}$ the proper transform of $V, V^{\prime}$ on the desingularizations $S, S^{\prime}$ of the minimal varieties $F, F^{\prime}$ containing $V, V^{\prime}$ respectively, if $k \leq r-2$. If $k=r-1$, we set $V=W, V^{\prime}=W^{\prime}$. Notice now that $U^{\prime}$ may be thought of as an open subset of $W^{\prime}$, and $\pi$ induces a birational morphism $\pi^{\prime}: U^{\prime} \rightarrow W$. Thus $C^{\prime} \subset U^{\prime}$ may be viewed as a curve in $W^{\prime}$ and, abusing notation, we shall denote again by $C$ the curve $\pi^{\prime}\left(C^{\prime}\right)$ in 
$W$. Consider now the two invertible sheaves $\omega_{W \mid C^{\prime}}, \omega_{W^{\prime} \mid C^{\prime}}$ on $C, C^{\prime}$ respectively, and let $d, d^{\prime}$ be their degrees. By Step 2 we clearly have

$$
d=d^{\prime} .
$$

Now we examine separately various cases.

Case 1: $k=r-1$. Here $\omega_{V}=O_{V}((n-r-1)), \omega_{V^{\prime}}=O_{V^{\prime}}((n-r-1))$. Thus $d=m(n-r-1), d^{\prime}=n(n-r-1)$, and since $n \geq r+2,(3.5 .1)$ yields $n=m$.

Case $2: k=r-2, V^{\prime}$ contained in a rank $\rho^{\prime} \geq 5$ quadric $F^{\prime}$. Then $n \equiv 0(\bmod 2)$ and formula (2.4.1) may be applied to $W^{\prime} . F$ is also a quadric with rank $\rho$. If $\rho \geq 5,(2.4 .1)$ holds for $W$ too. Then $d^{\prime}=n(n / 2-r+1), d \geq m(n / 2-r+1)$. Since $n \geq 2 r$, (3.5.1) implies $n=m$. If $\rho \leq 4, F$ is a scroll, and (2.3.3) holds, with $M=n / 2-1, e=1$, and again, like above, we find $n=m$.

Case 3: $k=r-4, V^{\prime}$ contained in a Veronese cone. According to results of $\S 2.5$, (2.5.1) applies to $W^{\prime}$, and there are three subcases to be discussed: (i) $n=4 a$, (ii) $n=4 a+4$, (iii) $n=4 a+2$. Assume $F$ is a scroll. Then formula (2.3.3) may be applied, since $M=a-1, e=3$ for (i) and (ii), while $M=a, e=1$ for (iii). Then in case (iii) one finds $n=m$ just arguing as before. By contrast, case (i) and (ii) lead to a contradiction. In fact $C^{\prime}$ is birational to a plane curve of degree $2 a$, and the same is true for $C$. Therefore, by (1.3.1), we have $R \cdot C \geq 2 a-1$. Finally

$$
\begin{gathered}
d^{\prime}=(a-r+3) H^{\prime} \cdot C^{\prime}+L^{\prime} \cdot C^{\prime}=4 a(a-r+3)+2 a, \\
d \geq(a-r+3) H \cdot C+2 R \cdot C \geq m(a-r+3)+4 a-2 .
\end{gathered}
$$

Since $a>r-3$, by (3.5.1) we should have $m<n=4 a$, and this is impossible (see (3.4.1)). At last, if $F$ is also a Veronese cone, it may easily be proved that $n=m$, by usual arguments.

Case 4: $V^{\prime}$ is contained in a scroll. The cases $F$ is not a scroll can be settled by changing the roles of $V$ and $V^{\prime}$, and applying the above discussion. So we may assume also $F$ is a scroll. If $e>0$ we may apply formula (2.3.3) in order to describe both $W, W^{\prime}$. By Theorem (1.5.1) we have $C \cdot R \geq C^{\prime} \cdot R^{\prime}$. Then $d^{\prime}=n(M-k)+(e-1) C^{\prime} \cdot R^{\prime}, d \geq m(M-k)+(e-1) C \cdot R$, and $n=m$ follows by (3.5.1) since $M \geq k+1$. If $e=0$ we have to discuss three subcases: (i) both $W, W^{\prime}$ are described by (2.3.3); (ii) $W$ is given by (2.3.4), $W^{\prime}$ by (2.3.3); (iii) both $W, W^{\prime}$ are described by (2.3.4). In the latter, again by (1.5.1), we have $C \cdot R \geq C^{\prime} \cdot R^{\prime}$, whence $n=m$ as above. In case (ii), by Theorem (1.5.1) we have

$$
C \cdot R \geq \begin{cases}M+1, & \text { if } k<r-2 . \\ M, & \text { in any case. }\end{cases}
$$

On the other hand $\left(H^{\prime}-R^{\prime}\right) \cdot C^{\prime}=M(r-k-1)$. Thus $d^{\prime}=n(M-k-1)+$ $M(r-k-1), d \geq m(M-k-1)+C \cdot R(r-k-1)$, which gives a contradiction if $k<r-2$, gives $n=m$ if $k=r-2$. Let us finally discuss case (i). The linear system $|H-R-E|$ cuts out on $C$ a base points free $g_{t}^{d}$. Suppose for a moment we have proved that $d \geq r-k-1$. Then Theorem (1.5.2) can be applied concluding that $t=(H-R-E) \cdot C \geq\left(H^{\prime}-R^{\prime}\right) \cdot C^{\prime}$, and this implies $n=m$. So we can finish Step 4 by proving the

(3.5.3) LemMA. The linear series cut out by $|H-R-E|$ on $C$ has dimension $d \geq r-k-1$. 
ProOF. We look at $C$ as a curve on $V$, spanning a $\mathbf{P}^{r-k+l}$, with $l \geq 1$. A general $\mathbf{P}^{r-k}$ in $\mathbf{P}^{r-k+l}$ can be assumed to be a general $\mathbf{P}^{r-k}$ in $\mathbf{P}^{r}$ (see the argument of $\S 3.4)$. This implies that $F_{0}=F \cap \mathbf{P}^{r-k+l}$ is an $(l+1)$-dimensional minimal scroll of $\mathbf{P}^{r-k+l}$. In fact any component of $F_{0}$ has dimension at least $l+1$. If its dimension were bigger, then its intersection with $\mathbf{P}^{r-k}$ would have dimension at least 2 . This is a contradiction, because, $\mathbf{P}^{r-k}$ being a general one, its intersection with $F$ is a rational normal curve. On the other hand, since $F_{0}$ contains $C$, it is also clear that $F_{0}$ is irreducible. Now the linear series cut out on $C$ by $|H-R-E|$ is also cut out on $C$ by all hyperplanes of $\mathbf{P}^{r-k+l}$ containing a $\mathbf{P}^{l}$ of the ruling of $F_{0}$. This proves the lemma.

3.4. Step 5: End of the proof. Let $D^{\prime}$ be a general hyperplane section of $V^{\prime}$ and let $D$ be the proper transform of $D^{\prime}$ on $V$ via $\pi$ : precisely the Zariski closure of $\pi\left(D^{\prime} \cap U^{\prime}\right)$. Theorem (3.1.1) will be proved by showing that $D$ is a hyperplane section of $V$. Since $D$ has degree $D \cdot C=D^{\prime} \cdot C^{\prime}=n$, it is enough to prove that $D$ cannot be nondegenerate. To this aim it is sufficient to remark that $D$ cuts a general curve section $C$ of $V$ with a $\mathbf{P}^{r-k+1}$ in $n$ points which do not span the $\mathbf{P}^{r-k+1}$ (see Remark (3.4.1)).

\section{REFERENCES}

[A] R. D. M. Accola, On Castelnuovo's inequality for algebraic curves. I, Trans. Amer. Math. Soc. 251 (1979), 357-373.

[H] J. Harris, A bound on the geometric genus of projective varieties, Ann. Scuola Norm. Sup. Pisa (4) 8 (1981), 35-68.

[HR] R. Hartshorne, Algebraic geometry, Graduate Texts in Math., Springer-Verlag, 1977.

[K] E. Kunz, Holomorphe Differentialformen auf algebraischen Varietäten mit Singularitäten. I, Manuscripta Math. 15 (1975), 91-108.

[R] M. Reid, Canonical 3-folds, Journées de Géométrie Algébrique d'Angers, Sijthoff \& Noordhoff, 1980.

[S] F. Severi, Sulle trasformate razionali di un'ipersuperficie algebrica priva di punti multipli, Math. Ann. 190 (1933), 4-6.

Dipartimento di MATEMATICA, II Università di Roma, Via O. Raimondo, 00173 ROMA, ITALY 